\title{
Jacques PARIZEAU
}

Professeurs d'économie, École des Hautes Études Commerciales, Montréal

(1960)

\section{"Les post-keynésiens et la politique économique contemporaine”}

Un document produit en version numérique par Jean-Marie Tremblay, bénévole, professeur de sociologie au Cégep de Chicoutimi

Courriel: jean-marie tremblay@uqac.ca

Site web pédagogique : http://www.uqac.ca/jmt-sociologue/

Dans le cadre de la collection: "Les classiques des sciences sociales"

Site web: http://classiques.uqac.ca/

Une collection développée en collaboration avec la Bibliothèque Paul-Émile-Boulet de l'Université du Québec à Chicoutimi

Site web: http://bibliotheque.uqac.ca/ 
Cette édition électronique a été réalisée par Jean-Marie Tremblay, bénévole, professeur de sociologie au Cégep de Chicoutimi à partir de :

Jacques Parizeau

Professeur d’économie, École des Hautes Études Commerciales, Montréal,

"Les post-keynésiens et la politique économique contemporaine"

Un article publié dans l'ouvrage de François-Albert ANGERS, ESSAI SUR LA CENTRALISATION. ANALYSE DES PRINCIPES ET PERSPECTIVES CANADIENNES. Deuxième partie, chapitre VII, pp. 291 à 313. Montréal : Les Presses de l’École des Hautes Études Commerciales et Les Éditions de la Librairie Beauchemin, 1960, 331 pp.

M Jacques Parizeau, économiste et ancien premier ministre du Québec, nous a accordé, le 18 septembre 2006 son autorisation de diffuser la totalité de ses publications. L’autorisation accordée au téléphone a été confirmée par écrit par M. Parizeau lui-même.

Polices de caractères utilisée :

Pour le texte: Times New Roman, 14 points.

Pour les citations : Times New Roman, 12 points.

Pour les notes de bas de page : Times New Roman, 12 points.

Édition électronique réalisée avec le traitement de textes Microsoft Word 2004 pour Macintosh.

Mise en page sur papier format

LETTRE (US letter), 8.5'’ x 11'’)

Édition numérique réalisée le 30 avril 2007 à Chicoutimi, Ville de Saguenay, province de Québec, Canada. 
Jacques Parizeau

Professeur d’économie, École des Hautes Études Commerciales, Montréal,
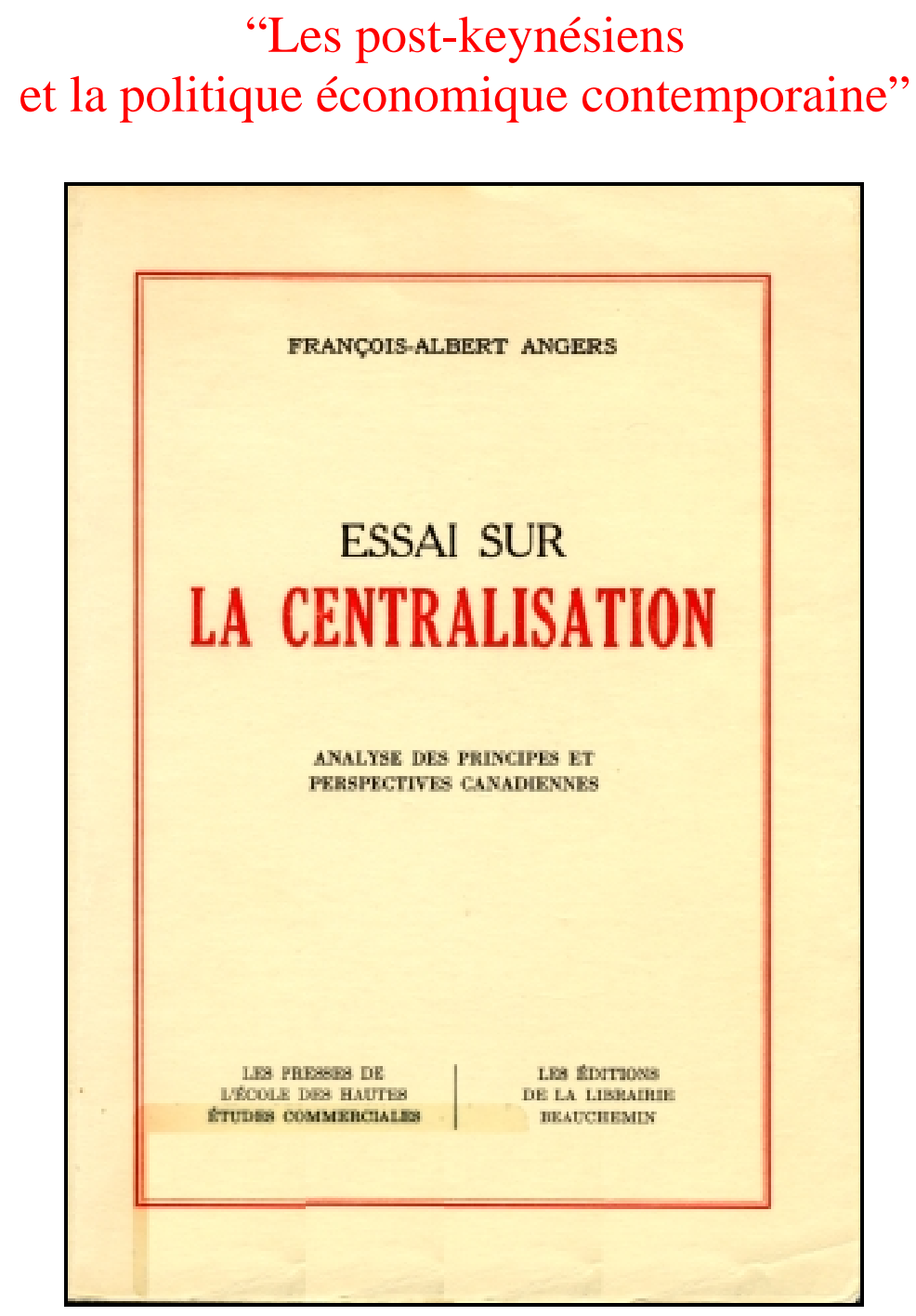

Un article publié dans l'ouvrage de François-Albert ANGERS, ESSAI SUR LA CENTRALISATION. ANALYSE DES PRINCIPES ET PERSPECTIVES CANADIENNES. Deuxième partie, chapitre VII, pp. 291 à 313. Montréal : Les Presses de l'École des Hautes Études Commerciales et Les Éditions de la Librairie Beauchemin, 1960, 331 pp. 


\title{
Table des matières
}

\author{
“Les post-keynésiens \\ et la politique économique contemporaine“. \\ Par Jacques Parizeau, \\ professeur à l'École des Hautes Études Commerciales de Montréal
}

1. Prolongements et transformations de la théorie keynésienne : la Théorie Générale du financement de la guerre sans inflation - fiscalité et politique économique : effets des travaux empiriques sur l'évolution de la théorie les spécifications outrancières

2. À la recherche d'une politique applicable : les difficultés d'application d'un programme anticipé - les difficultés de prévisions des effets - l'adultération de la théorie au contrôle de la pratique

3. Les post-keynésiens et l'économie internationale: les travaux de Joan Robinson - les études empiriques et leur conclusion sur l'élasticité - la fin des illusions : Orcutt

4. Le retour au classicisme monétaire 


\title{
"Les post-keynésiens et la politique économique contemporaine”
}

\author{
par \\ Jacques Parizeau \\ Professeur d'économie \\ à l'École des Hautes Études commerciales \\ de Montréal
}

La théorie keynésienne avait à peine été diffusée dans les pays anglo-saxons que les conditions historiques qui étaient largement responsables de son élaboration et de son succès disparaissaient. Dès 1940, des pressions inflationnistes commençaient à se manifester un peu partout dans le monde et le niveau de l'emploi montait rapidement. Dans les années qui suivirent, le plein emploi était réalisé dans les pays industriels et le climat de pessimisme généralisé qui avait assuré la vogue des idées keynésiennes évoluait vers un optimisme à court terme qui, pour la durée de la guerre, tout au moins, endormait les craintes et les appréhensions. Sans doute certains économistes prévoyaient-ils la réapparition du chômage après la cessation des hostilités ; de nombreux ouvrages écrits à la fin de la guerre suggéraient les mesures inspirées d'un keynésianisme orthodoxe pour faire face à une crise analogue à celle qui avait suivi la première Grande Guerre, en 1921 et $1922 .{ }^{1}$ En principe la théorie du sous emploi de Keynes, et

1 Les prévisions des économistes, en temps de guerre, furent très partagées. Certains écrivains, comparant la crise qui a suivi la premier Grande Guerre à 
son prolongement, la théorie de la maturité économique développée par des disciples américains, restaient acceptées dans de nombreux milieux. La situation créée par la guerre était considérée comme un phénomène exceptionnel. Vienne la démobilisation et la théorie redeviendra valable. La démobilisation est venue et la dépression annoncée ne s'est pas produite. Un léger ralentissement, correspondant à la reconversion des usines a été suivi d'une période d'inflation intense qui n'a commencé à faiblir qu'à la fin de 1948. Les prévisions s'en trouvèrent alors changées. Si la dépression ne s'était pas produite c'est que l'arriéré de la demande de biens de consommation était considérable. Normalement la dépression devait se produire lorsque l'arriéré disparaîtrait. La dépression serait d'autant plus forte que les investissements opérés dans les années d'après-guerre servaient à satisfaire une demande anormale. 2

La dépression se produisit en 1949. Mais elle n'avait pas la force que l'on avait prévue ; après une récession de quelques mois, une stabilisation était intervenue qui semblait bien devoir être suivie d'une reprise lorsque la guerre de Corée intervint. Une nouvelle hausse des prix se produisit. Les prix de certaines matières premières, sous la double impulsion d'une accélération de l'activité économique et de la politique de stockage du gouvernement américain, montèrent rapidement à la fin de 1950 et au début de 1951. Cette fois il semblait y avoir de bonnes raisons pour croire qu'une fois la guerre terminée, le relâchement de l'effort militaire des États-Unis amènerait une récession soudaine. En fait, malgré la chute rapide des prix de certaines matières premières sur le marché mondial, la récession ne fut guère sensible aux États-Unis qu'en 1954, et encore faut-il remarquer que plusieurs pays d'Europe ne furent pas touchés par cette récession.

la seconde semblaient assurés que la crise viendrait rapidement et serait sévère, à moins que des mesures urgentes ne soient prises. On lira, parmi bien d'autres articles, celui de Samuelson "Full Employment after the War", dans Postwar Economic Problem, édité par S.-E. Harris, McGraw-Hill, New-York, 1943. Après avoir dégagé les différences qui existent entre les deux économies de guerre, Samuelson est particulièrement pessimiste.

2 Hansen, dans une note publiée dans Saving American Capitalism (édité par S.E. Harris, Alfred-A. Knopf, New-York, 1948), pp. 218 et s., entrevoit un approfondissement des mouvements cycliques d'investissements. Il avait été assez circonspect dans ses prédictions au cours de la guerre. 
Dans bien des pays, le plein emploi était maintenu et dix ans après la guerre, le rythme de croissance des économies se maintenait à un haut niveau.

En somme, les économistes se trouvent placés, depuis la fin de la deuxième Grande Guerre, dans un climat psychologique tout à fait différent de celui qui avait marqué l'élaboration des idées keynésiennes. On ne peut s'imaginer que le changement de climat n'ait pas influencé le développement de la théorie et n'ait pas, par surcroît, amené la révision de certaines des idées fondamentales acceptées à une autre époque. D'une façon plus précise, la combinaison d'une inflation intermittente et généralisée, coupée de récessions courtes et de faible amplitude qui font figure de paliers plutôt que de chutes véritables, et de la pérennité d'un haut niveau de l'emploi, ont suffisamment impressionné les techniciens pour que les plus dogmatiques d'entre eux aient été attirés par l'étude de phénomènes qui n'avaient été qu'effleurés par Keynes et aient été forcés de réviser ce qui dans la théorie générale n'avait qu'une valeur épisodique. D'autres économistes, enfin, ont cru nécessaire de modifier les éléments fondamentaux de la théorie keynésienne.

Le texte qui suit ne peut pas être une rétrospective même schématique du développement des idées et des principes d'action des économistes qu'on désigne aujourd'hui sous le vocable, d'ailleurs très lâche, de post-keynésiens. Le but de l'auteur est seulement de chercher à dégager quelques-unes des transformations ou des prolongements les plus caractéristiques de la théorie keynésienne, dans la mesure où ils affectent l'orientation de la politique économique ou, d'une façon plus générale, le climat dans lequel les politiques économiques tirent souvent sinon leur source principale, du moins leur coloration. 


\section{Prolongements et transformations de la théorie keynésienne}

$\underline{\text { Retour à la table des matières }}$

Il est assez difficile de déterminer avec quelque précision ce qu'il faut entendre par une position théorique post-keynésienne. Peu d'économistes n'ont pas été influencés fortement par des idées de Keynes. Les instruments d'analyse qu'il a forgés, les expressions, les termes qu'il a lancés ou propagés sont utilisés par bon nombre d'économistes dont la pensée évolue aux antipodes de l'orthodoxie keynésienne ou sont en contradiction flagrante avec les descendants spirituels directs de Keynes. D'ailleurs, sous l'influence des événements récents, d'idées nouvelles, et des résultats obtenus grâce à des études empiriques plus nombreuses, les positions théoriques contemporaines sont sûrement plus dispersées qu'elles ne l'étaient il y a dix ans. Dans certaines branches de l'économie, les oppositions sont même devenues si fréquentes et si poussées que l'on peut aisément parler d'un éclatement d'un corps de doctrine et d'une situation confuse où bien des principes acceptés sont remis en question. La politique économique des États s'est vivement ressentie de cet état de chose.

Le renversement des préoccupations des économistes à l'occasion des années d'inflation a été amorcé par Keynes qui a, une fois de plus, ouvert la voie à l'élaboration d'idées nouvelles. Dans son livre How to Pay for the $\mathrm{War}^{3}$, il lance ce qui sera la première de nombreuses études de ce qu'il est maintenant convenu d'appeler l'écart inflationniste (inflationary gap).

Les analyses de l'inflation qui suivirent furent essentiellement conduites à partir des concepts simples et globaux utilises par les keynésiens depuis l'apparition de la Théorie Générale. L'écart entre le revenu national et la consommation « autonome », c'est-à-dire celle

3 J.-M. Keynes, How to pay for the war, MacMillan, Londres, 1940. 
qui serait justifiée par la propension à consommer normale, ne serait pas suffisant pour couvrir les dépenses de guerre. Il est donc nécessaire de diminuer cette propension normale par une politique fiscale qui réduit la consommation au niveau désiré, c'est-à-dire à celui qui est compatible avec les investissements et les dépenses d'armement rendues nécessaires par la guerre et l'accroissement de la capacité des usines. 4

Sans doute toute l'analyse de l'écart inflationniste peut être faite à partir des méthodes que l'on convient de qualifier de keynésiennes. Il reste cependant que la théorie originelle a reçu dans ce contexte historique de profondes entorses. En effet, les expériences de la guerre et de l'après-guerre ont bien montré que la stabilité de la propension à consommer, dont on connaît l'importance dans le système de la Théorie Générale, dépendait de la stabilité d'un grand nombre de facteurs. C'est ainsi, par exemple, qu'au moyen d'une modification du régime de la fiscalité on a pu facilement transformer en variables ce que les keynésiens orthodoxes avaient pu déjà considérer, en temps normal, pour des quasi-constantes. Déjà Keynes avait souligné qu'une des façons de réduire une épargne surabondante pour éviter le retour à un équilibre de sous emploi aurait consisté à rendre très fortement progressifs les taux d'impôts sur les revenus de façon à diminuer les revenus élevés des groupes qui épargnent beaucoup pour accroître les revenus des classes qui consomment la plus grande partie de ce qui leur est versé. 5

Le contrôle de l'économie au moyen de la fiscalité a connu une vogue considérable pendant et immédiatement après la guerre. Hansen et son école aux États-Unis n'ont pas peu contribué à répandre une formule et un programme. ${ }^{6}$ De ces espoirs soulevés par les manipula-

4 Parmi les nombreux auteurs qui ont étudié l'écart inflationniste de cette façon, on remarque en particulier L.-R. Klein, The Keynesian Revolution, MacMillan, Londres, 1950.

5 J.-M. Keynes, The General Theory of Employment, Interest and Money, MacMillan and Co., London 1949, pp. 324-325. [Texte disponible, en version française, dans Les Classiques des sciences sociales. JMT.]

6 L'ouvrage, peut-être le plus marquant dans cette veine, et un de ceux qui ont eu une influence profonde par la suite, est celui de A.-H. Hansen. Fiscal Policy and Business Cycles, Norton \& Co., New-York, 1941, pp. 298-300. 
tions de la politique fiscale, on a pu dire qu'elles avaient été à l'origine du projet de stabilisation de l'emploi de 1946 aux Etats-Unis (Employment Act). ${ }^{7}$

Il est cependant devenu de plus en plus clair, à la longue, qu'une analyse des pressions inflationnistes ou déflationnistes qui étaient axées sur la politique fiscale seulement masquait certains phénomènes importants qui n'avaient pas été retenus par le Keynes de la Théorie Générale comme étant particulièrement importants. En effet, l'inflation contrôlée des années de guerre et l'inflation ouverte qui les a suivies a attiré l'attention, avec une insistance croissante, sur les aspects dynamiques du circuit économique. Le système keynésien, comme on l'a noté précédemment, est un système statique en ce sens qu'il n'est basé que sur des états successifs de la conjoncture sans décrire les périples et les caractéristiques des transformations qui séparent un état d'un autre. 8

On s'est rapidement rendu compte que dans une conjoncture explosive, le périple d'une situation est largement déterminé par les situations précédentes. On en est donc petit à petit arrivé à une analyse dynamique. Déjà Keynes, malgré des réticences anciennes, avait indiqué que les décalages d'ajustement (lags) avaient une influence déterminante sur l'intensité des forces inflationnistes. Ce décalage est, en somme, l'écart de temps qui sépare les diverses étapes de la circulation des revenus. C'est ainsi, par exemple, qu'un écart de temps sépare la consommation à même un revenu, de l'acquisition de ce revenu. L'écart peut être plus ou moins long et il est certain que la longueur de l'écart déterminera partiellement l'intensité de la pression exercée par la consommation sur les prix lorsque les revenus s'accroissent rapidement. Un écart très long impliquerait qu'à un moment donné la consommation réelle soit bien inférieure à la consommation corres-

7 A Survey of Contemporary Economics, édité par Howard-S. Ellis, Blakiston Co., Philadelphie, 1949, p. 176.

8 Voir précédemment deuxième partie, chapitre I. Au sujet du rôle de la théorie statique de Keynes sur le développement d'une dynamique théorique, on peut consulter le livre de J.-A. Schumpeter, History of Economic Analysis, Oxford University Press, 1954, pp. 1183-1184. 
pondant au revenu perçu au même moment. Les prix se seraient donc accrus moins rapidement que les revenus.

Des décalages de toutes sortes interviennent dans l'étude d'une situation économique et les économistes ou les économètres ont cherché à dégager le comportement des facteurs que Keynes avait isolés. ${ }^{9}$ Dans ce contexte, la théorie originale perdait déjà un peu de sa précision et de son élégance. En tout cas, elle atteignait à un caractère dynamique qui s'est largement étendu depuis.

On doit cependant noter que les premiers travaux empiriques élaborés à partir des concepts keynésiens avaient préservé l'essentiel du cadre théorique. Sans doute, comme il a été indiqué plus haut, des aménagements avaient dû être faits pour tenir compte de l'influence de la politique de l'État mais, dans l'ensemble le cadre tenait encore. Plus récemment, des travaux d'ordre empirique plus précis ont encore contribué à jeter des doutes sur des éléments beaucoup plus fondamentaux. L'étude de ce qu'il est convenu d'appeler la fonction de consommation a donné récemment des résultats imprévus. On a observé, par exemple, que la propension à consommer semblait avoir été très instable depuis quelques années, sans qu'on puisse attribuer cette instabilité exclusivement à une réorientation de l'action de l'État. L'inflation, en effet, redonnait aux anticipations des consommateurs, un rôle qu'on ne leur avait pas toujours assigné.

On risque même d'en arriver à une position abusive. Ayant longtemps cru à la stabilité de la fonction de consommation, certains économistes sont peut-être en voie de la considérer comme une fonction assez capricieuse. 10

Le peu d'importance que l'on accorde depuis quelques années aux relations « normales » ou « naturelles » de Keynes a d'ailleurs une au-

9 Les méthodes utilisées pour ce genre d'étude ont été très fortement influencées par le travail de Jan Tinbergen, Statistical Testing of Business Cycle Theory (2 vol.), Société des Nations, Genève, 1939. Le livre de Tinbergen reste un des classiques du genre.

10 A.-F. Burns, The Instability of Consumer Spending, 32nd Annual Report, National Bureau of Economic Research, mai 1952, p. 9. 
tre origine. La statique keynésienne considérait les phénomènes économiques comme des éléments induits. En d'autres termes, il était admis que l'apparition d'un équilibre de sous emploi n'est concevable que dans la mesure où des investissements de caractère spontané ne sont pas entrepris sur une vaste échelle. Les transformations industrielles, les soubresauts militaires ou politiques qui, brutalement ou progressivement, ouvrent à l'investissement des possibilités jusque-là ignorées étaient considérés comme des phénomènes peu fréquents, hasardeux, qui ne changeaient pas la validité permanente des nouvelles lois de l'économie, même si, en raison de ces Perturbations, les lois devaient parfois souffrir des exceptions. Dans le climat psychologique des années 1930 cette position s'entendait bien et était faite pour servir de préambule aux théories de la maturité économique. La guerre et l'après-guerre cependant, en changeant le climat, attirent l'attention sur l'importance des perturbations du système quant au caractère spontané de certains types d'investissements. Sans doute les guerres sont-elles des accidents, les découvertes technologiques sont-elles irrégulières, et l'apparition de nouvelles couches de besoins est-elle imprévisible. Mais on se rend compte que si un système statique peut à la rigueur exclure ces accidents, un système dynamique ne peut les éliminer tous et que de toute façon, ces accidents ont été suffisamment nombreux dans le passé pour rendre variable la relation de l'investissement et du revenu. Alors même que dans le cadre d'une théorie statique, l'épargne, en fonction d'un revenu donné, devrait être supérieure à l'investissement prévu, le courant dynamique de l'économie implique souvent qu'une perturbation ou un développement des possibilités d'investissements rétablira l'équilibre. ${ }^{11}$

Cet assouplissement du système keynésien a été facilité par l'étude de plus en plus poussée des relations techniques qui relient un volume donné d'investissements à la génération des revenus. Ces études, rendues possibles par l'amélioration considérable des statistiques, ont amené les théoriciens à ne pas considérer a priori comme constantes, les variations de la relation de l'investissement et du revenu. Dans le temps, ce genre de variation peut avoir une influence très nette sur la

11 Une critique pondérée de la théorie post-keynésienne des possibilités d'investissements a été publiée par D.-M.C. Wright, dans le Survey of Contemporary Economics, op. cit., pp. 449 et s. 
génération des revenus et leur distribution. Il est donc difficile de conclure à la validité d'une loi générale des comportements des investissements, comme l'ont fait certains post-keynésiens. ${ }^{12}$

En somme, sous la double influence des faits et des idées, une transformation s'est opérée à l'intérieur de la théorie keynésienne. En remplaçant les relations quasi constantes qui caractérisaient la théorie par des relations variables, c'est-à-dire en constatant que les dispositions à la consommation ou à l'investissement des individus dans une nation peuvent facilement ou bien se transformer d'elles-mêmes ou bien encore être considérablement modifiées, ou enfin, ne pas toujours avoir les mêmes effets, on a retiré à la théorie keynésienne une bonne partie de ce qui faisait sa force, sa généralité et son intérêt. On peut même en conclure, que dans le cas de bon nombre d'économistes, les éléments essentiels de la position de Keynes ne sont plus acceptés.

Cependant que la théorie keynésienne était attaquée dans ses fondements et à partir de ses propres données, le travail de sape se poursuivait de l'extérieur, c'est-à-dire à partir de notions qui avaient été laissées dans l'ombre par Keynes ou plutôt par ses disciples. Il faut dire d'ailleurs que certains keynésiens en étaient arrivés dans les années qui précèdent la guerre à une incroyable insouciance de tout ce qui n'était pas directement relié aux quantités globales utilisées par le nouveau système. La méconnaissance du comportement des prix affichée par certains keynésiens était devenue pénible. La citation suivante tirée d'un livre qui a eu un profond retentissement est, de ce point de vue, caractéristique : «Dans tous les modèles élaborés au

12 L'étude des relations de l'investissement et du revenu a été entreprise simultanément par un bon nombre d'économistes et pour des raisons tout à fait différentes. W. Leontieff, dans ses tables d'input-output, a rapidement conclu aux difficultés qu'il y a de poser comme hypothèse des relations techniques stables entre une quantité de facteurs de productions et une quantité de produits finis. W. Leontieff, The Structure of American Economy, 1919-1939, Oxford University Press, New-York, 1950, pp. 214 et s. Les recherches entreprises au sujet de l'industrialisation des pays sous-développés ont aussi fait apparaître la nécessité de tenir compte des variations des coefficients techniques de production. 
cours de cette étude, on a posé l'hypothèse... que les prix (y compris les taux de change et les taux d'intérêt) sont... stables ». 13

Les post-keynésiens (et il en reste encore actuellement) qui pratiquent ce genre de chirurgie dans le complexe de la vie économique ne sont pas nécessairement des rêveurs. Nombreux sont ceux qui considèrent les liaisons de quantités globales comme une approximation valable de la vie économique, et il n'est pas démontré que dans le cas de certains types de conjoncture, ils n'aient pas raison.

Il reste cependant que, dans bon nombre de champs théoriques et, en particulier dans celui du commerce international, les postkeynésiens ont souvent procédé à des simplifications outrancières et à des suppositions qui n'avaient plus rien de commun avec la théorie de Keynes. 14

Dans la conjoncture économique de l'après-guerre, plusieurs de ces hypothèses simplificatrices ne correspondaient plus à aucune réalité. Supposer que l'élasticité de l'offre était infinie n'avait plus de sens, ou du moins, plus d'utilité. La rigidité des salaires devenait un concept dépassé par les événements. Enfin, ne considérer des prix que leur niveau général risquait de mener à des conclusions trop approximatives. En effet, l'inflation des années récentes, en provoquant un bouleversement des relations des prix les uns par rapport aux autres, attira une fois de plus l'attention sur l'importance des mouvements individuels.

Cette orientation nouvelle se comprend d'autant mieux que les disparités de prix étaient associées à un morcellement du marché de l'emploi et de la capacité de production. Cela demande quelques développements. A une époque de sous emploi chronique, il est tout-à-fait admissible de supposer que si l'emploi s'accroît soudainement, l'effet s'en fera sentir sur l'ensemble du marché de l'emploi. De même, si les prix et la production montent, on peut supposer que tous les secteurs de l'économie réagiront de la même façon. En d'autres termes, les ni-

13 Fritz Machlup, International Trade and the National Income Multiplier, Blakiston Company, Philadelphie, 1950, p. 19.

14 Le cas spécial des applications de la théorie keynésienne au commerce international et à la politique commerciale est traité à la fin de ce chapitre. 
veaux de l'emploi des prix et de la Production sont des réalités valables et relativement homogènes.

Cependant, au fur et à mesure que s'intensifie l'activité économique, des goulots d'étranglement apparaissent dans certains secteurs, c'est-à-dire qu'en raison du bas niveau relatif de la capacité de production dans ces secteurs, la production ne peut être accrue facilement. Dans d'autres secteurs, au contraire, la production reste très élastique. Dans ces conditions, les disparités de prix et de salaires deviennent considérables, d'un secteur à un autre, et les concepts globaux et généraux, valables dans le cas d'un sous-emploi généralisé, le sont de moins en moins dans la nouvelle conjoncture.

La situation inflationniste des années récentes a donc suscité un renouvellement des études empiriques du marché qui avaient été en vogue au cours des années qui ont précédé la Théorie Générale. Les méthodes, sans doute, étaient différentes de ce qu'elles étaient à cette époque, mais le souci d'observation précise et de théorie nuancée, était analogue. Sans doute, les post-keynésiens n'ont pas été à l'avant-garde de ce mouvement qui s'est développé en marge de leurs préoccupations principales. Mais le renouveau d'intérêt dans le sens de l'étude parcellaire du marché n'a pas peu contribué à donner une prudence extrême à des conclusions qui, il y a encore quelques années, s'étalaient dans toute leur simplicité.

\section{2. À la recherche d'une politique applicable}

$\underline{\text { Retour à la table des matières }}$

Quelle que soit l'origine des influences qui, de l'intérieur ou de l'extérieur, ont transformé la théorie qu'il est convenu d'appeler keynésienne, elles ont été suffisamment puissantes pour enlever beaucoup d'assurance et de précision à la politique économique suggérée par les post-keynésiens. Les tentatives d'application des principes orthodoxes et d'une façon plus générale, les politiques économiques pratiquées 
depuis une quinzaine d'années, ont provoqué des modifications des principes directeurs de l'action de l'État sur la conjoncture. Trois de ces modifications paraissent avoir été particulièrement importantes : la première a trait à la dynamique de la politique économique keynésienne ; la seconde, à son caractère global ; la troisième à ses répercussions à long terme.

Après avoir suggéré une série de mesures, les économistes n'ont pas pris de temps à se rendre compte que les grands travaux, les mesures de sécurité sociale et d'une façon générale les diverses méthodes prévues pour maintenir et élever le niveau de l'activité économique et, de l'investissement étaient peut-être valables mais n'avaient pas toutes les mêmes effets et que ces effets ne se produisaient pas toujours dans un même espace de temps. Dès les années d'après-guerre, les postkeynésiens, ou du moins certains d'entre eux, avaient commencé à s'intéresser à la mise en place et à l'organisation systématique d'une politique anticyclique qui n'était plus conçue en termes strictement conceptuels mais devait être intégrée à l'organisation administrative nationale et au circuit des revenus. L'organisation du Public Works Reserve, aux États-Unis, marque une première étape dans cette adaptation de la politique économique à la vie économique de la nation dans ce qu'elle peut avoir de dynamique et de complexe. ${ }^{15}$

En plaçant ainsi leurs propositions sur un terrain essentiellement pratique, les post-keynésiens constatèrent un certain nombre de difficultés imprévues qui, sans changer l'espoir que l'on avait mis dans la nouvelle politique économique, rendaient souvent impératives des réformes -de structures. En effet, il est devenu rapidement évident que, dans la vie réelle, un programme anticyclique ne pouvait être mis en place sans de longs détours. En Amérique du Nord, en particulier, les plans de grands travaux exigent une préparation administrative à trois échelons gouvernementaux, et une collaboration incessante des échelons. La mise en place d'une législation sous un régime parlementaire et fédéral demande des délais de plusieurs mois sinon de quelques années.

15 Voir à ce sujet Problems of Planning Public Works de Benjamin Higgins dans "Postwar Economic Problems", McGraw-Hill Co., NewYork, 1943. 
On semble donc avoir le choix, soit d'attendre qu'une dépression se soit déclarée et ait pris de l'ampleur avant de préparer la politique anticyclique mais alors on doit revenir de loin, soit de lancer le programme dès qu'un fléchissement se dessine, mais alors les effets de ce programme risquent de se produire à contretemps - Si dans l'intervalle l'économie s'est raffermie - et de jouer dans le sens de l'inflation. L'alternative n'a rien de didactique. Au cours des légers fléchissements des dix années qui ont suivi la guerre, il est arrivé que des mesures qui devaient combattre le fléchissement se sont exercées sur une conjoncture en hausse.

Ces contestations ont amené les post-keynésiens à recommander une centralisation des pouvoirs gouvernementaux et, à reconnaître l'utilité de certains contrôles directs sur l'économie. ${ }^{16}$ D'un point de vue de stricte efficacité technique une centralisation aussi étendue que possible permettrait, selon eux, de redonner au programme anticyclique la flexibilité qui est rendue nécessaire par la complexité de l'économie. Cette tendance à la remise de pouvoirs de plus en plus étendus à l'État reste compatible avec l'orthodoxie théorique.

D'un autre point de vue, cependant, la politique économique postkeynésienne semble devoir rompre avec ses attaches. En effet, il est devenu de plus en plus clair que les instruments mis à la disposition de l'État par les théoriciens ont une influence infiniment moins prévisible qu'on l'avait d'abord cru. En un certain sens, le retour des théoriciens vers la micro-économie, et la fragmentation des quantités globales que l'on concevait à l'origine comme étant relativement homogènes, ont été accompagnés d'une fragmentation de la politique économique. Les exemples sont nombreux, mais sont particulièrement nets dans le domaine de la fiscalité. Les post-keynésiens ont accordé un rôle très important à l'impôt sur le revenu par opposition aux impôts indirects. ${ }^{17}$ Cela se comprend aisément. L'impôt direct semble être

16 A. Smithies, "The Work and Influence of John Maynard Keynes", dans le Quarterly Journal of Economics, novembre 1951, p. 599.

17 Hansen, par exemple, suggérait en 1943 que la politique fiscale des années d'après-guerre devrait conserver une structure d'impôts sur les revenus élevés mais réduire les taxes de ventes imposées pendant la guerre. Postwar Economic Problems, op. cit., p. 22. D'une façon générale, les keynésiens considèrent 
éminemment susceptible d'élever rapidement la propension marginale à consommer. C'est un effet clair et précis.

En pratique cependant, l'impôt sur le revenu, élevé comme il l'est depuis la guerre a des effets imprévus. Il renforce considérablement, par exemple, la puissance relative des monopoles et des oligopoles, et force l'État à un surcroît de vigilance pour éviter l'expansion du contrôle des marchés. ${ }^{18}$ D'autre part, un impôt direct très lourd risque souvent de nuire à la croissance, soit d'un secteur de l'économie soit de l'économie dans son ensemble. Comme il est difficile de maintenir des taux d'imposition fortement différentiels, un taux élevé peut en arriver à museler l'expansion de certains secteurs. Enfin, et surtout, l'impôt direct ne permet guère de contrôler avec précision le niveau de la consommation. Dans le cadre théorique du circuit des revenus, l'impôt direct doit servir en temps d'inflation à limiter le pouvoir d'achat. Étant donné, cependant, l'élargissement de l'éventail des prix, l'existence de goulots d'étranglement dans certains secteurs et d'une capacité de production inemployée dans d'autres, les variations du taux de l'impôt direct semblent être un moyen un peu gros d'orienter la consommation en fonction de la quantité disponible de chacun des produits. La ponction opérée dans les revenus peut faire illusion sur l'efficacité du programme. Elle n'atténue pas le désordre du marché.

En d'autres termes, la fiscalité des post-keynésiens apparaît de plus en plus comme étant un outil peu précis dont les effets étaient prévisibles dans un système théorique où les variables étaient peu nombreuses, mais nettement moins prévisibles dans la conjoncture réelle. Pendant quelque temps, on a semblé chercher du côté des impôts indirects et en particulier de la taxe de vente, la flexibilité et la précision nécessaires aux réalités économiques de notre temps. ${ }^{19}$ Cela correspond

les impôts directs progressifs comme les plus susceptibles de jouer dans le sens d'une réduction de l'épargne.

18 Inter alia, Fritz Machlup, The Political Economy of Monopoly, John Hopkins, Baltimore, 1952, pp. 250 et s.

19 Hansen avait déjà préparé ce revirement. "Thus, for a dynamic, expanding economy enjoying vigorous booms a fluctuating consumption tax may be the appropriate tax policy; for a less rapidly expanding, mature economy a fixed but steeply progressive income tax structure is indicated." Fiscal Policy and Business Cycles, op. cit., p. 300. 
d'ailleurs aux responsabilités que l'État acquiert d'orienter la croissance de la nation. La croissance n'est pas une quantité globale, ou du moins n'est pas caractérisée par la belle simplicité de la macroéconomie. l'État doit pouvoir encourager un secteur, en décourager un autre, diriger la production, et pour ce faire a besoin d'une technique dont on perçoit dès maintenant les premiers tâtonnements. Cette technique garde, sans doute, de la théorie keynésienne certains de ses concepts et certains de ses postulats. Il devrait être clair, après ce qui a été signalé plus haut, que le moule n'est plus le même.

Si les post-keynésiens éprouvent des difficultés à faire passer leurs idées dans le cadre pratique de la politique actuelle, ils ont aussi des difficultés particulières à préserver la pureté de leur doctrine. Il semble bien, en effet, que le succès particulier de certaines mesures proposées par les keynésiens, depuis la guerre, a été assuré moins par la conviction des gouvernements vis-à-vis la théorie de Keynes ou de ses disciples que par la puissance des régimes socialistes dans divers Pays du monde après 1945. Sans doute, les programmes de sécurité sociale sont-ils conformes aux idées des post-keynésiens dans la mesure où, en accroissant la propension à épargner, ils retardent l'échéance d'une surabondance d'épargne. Encore faut-il voir que ce principe, s'il donne une justification économique de la sécurité sociale, n'est guère qu'une excuse, utile sans doute, mais qui n'est pas une condition nécessaire à la mise en place du programme.

En un sens, l'expansion de la sécurité sociale a fait illusion. Certains ont vu dans cette expansion la mise en œuvre des idées keynésiennes, sans toujours noter qu'il y avait une opposition flagrante entre l'administration réelle de ces programmes et l'administration suggérée par les keynésiens. La sécurité sociale, en effet, telle qu'elle a été appliquée en Europe, en particulier, est devenue une charge sociale rigide. Il ne peut pas être question, actuellement, pour des raisons psychologiques et politiques, de donner une grande flexibilité aux versements et aux indemnités. Réduire ces indemnités en cours d'inflation, est sûrement impossible ; les accroître en temps de récession est rendu d'autant plus difficile qu'en raison de l'enthousiasme initial, les charges budgétaires sont très lourdes et peu susceptibles d'être accrues, sur la courte période, tout au moins. Il n'y a donc rien de particulièrement anti-cyclique dans les politiques contemporaines de sécurité sociale. 
Dans la conjoncture inflationniste d'après-guerre, les mesures qui ont été prises n'ont fait qu'accentuer les pressions à la hausse de prix, sans qu'on puisse affirmer que sans ces mesures, le rythme d'accroissement des investissements aurait beaucoup varié.

Il ne s'agit pourtant pas de condamner ici les applications qui ont été faites de la sécurité sociale, sous prétexte qu'elles ont nui à la stabilité des prix. On veut plus simplement indiquer que ces applications ont eu, en somme, peu de points de contact avec les post-keynésiens et puisent leur origine doctrinale dans une large mesure en dehors du cadre keynésien. Le rapport Beveridge est, à sa source, beaucoup plus l'expression des principes fabiens que celle de la théorie de Keynes.

On peut donc conclure, après ces vues succinctes sur la politique post-keynésienne que, dans des perspectives à long terme, elle se fond dans un ensemble de principes plus ou moins abstraits et de pratiques imposées par le déroulement des événements, qui ont perdu et perdent de plus en plus les attaches qu'ils semblaient avoir, à la fin de la guerre, avec la Théorie Générale. On était assuré, il y a dix ans, de centrer la politique économique dans le cadre keynésien. On est beaucoup moins sûr de nos jours que le centre de gravité politique ne soit pas très éloigné du point de départ qu'on lui avait fixé.

\section{Les post-keynésiens et l'économie internationale}

$\underline{\text { Retour à la table des matières }}$

jusqu'ici, la théorie et la politique keynésiennes n'ont pas été abordées sous l'angle des relations internationales. C'est en un certain sens commettre une injustice à l'égard des post-keynésiens que de passer maintenant au commerce extérieur, puisque, plus que tout autre groupe d'économistes, ils ont contribué à l'intégration analytique du secteur intérieur et du secteur extérieur de l'économie. L'étude des idées post-keynésiennes sur les balances des paiements doit cependant être traitée à part, du point de vue qui est soulevé dans ce texte. La 
théorie du commerce international a, en effet, été marquée par des revirements profonds depuis quinze ans et ses détours sinueux illustrent, d'une façon exagérée sans doute, certaines des idées qui ont été présentées dans les pages qui précèdent.

Keynes avait, en somme, peu touché au commerce extérieur. Préoccupé par les conditions internes d'une économie déprimée, il s'était contenté de faire ressortir les forces qui déterminent directement le niveau de l'emploi. La consommation, par exemple, n'est pas à proprement parler nationale chez Keynes ; il n'y a pas de distinction nette entre la demande nationale effective et la demande internationale effective. 20

Bien entendu, les premiers économistes américains qui ont adopté les principes de la Théorie Générale n'étaient pas non plus très attirés par l'économie internationale, étant donné la faiblesse relative du commerce extérieur américain et l'énormité des mouvements de la conjoncture aux États-Unis.

Cependant, en marge du courant principal, certains économistes keynésiens, et parmi les premiers, étendirent la théorie originale au secteur de l'économie internationale, après quelques adaptations. Un des premiers et des plus importants des textes parus sur cette question est le livre de Joan Robinson : Essays in the Theory of Employment. La troisième partie de cet ouvrage porte sur les taux de change et les politiques d'exportation du chômage. ${ }^{21}$ Là se trouve la source de très nombreux développements. Tous ne seront pas abordés ici, et ce qui suit doit être interprété comme une indication de certains des traits caractéristiques de ce qu'on a appelé la nouvelle théorie du commerce international.

L'équilibre intérieur keynésien était entièrement déterminé à partir d'un équilibre de quantités globales, en d'autres termes, à partir d'un

20 «... the effective demand is simply the aggregate income (or proceeds) which the entrepreneurs expect to receive..." J.-M. Keynes, The General Theory, etc., op. cit., p. 55.

21 Joan Robinson, Essays in the Theory of Employment, Basil Blackwell, Oxford, deuxième édition 1947, pp. 134-170. 
équilibre macro-économique. Le même principe a été transposé dans le domaine du commerce extérieur.

Dans le système classique, l'équilibre de la balance des comptes était déterminé par les prix : en effet, des excès d'exportations provoquaient une entrée de capitaux qui à son tour provoquait une hausse des prix, qui décourageait les exportations. Le même mécanisme appliqué en sens inverse dans le pays débiteur accélérait le retour à l'équilibre.

Dans une large mesure les post-keynésiens se débarrassent des mouvements des prix comme facteurs d'équilibre. Si pour une raison quelconque un pays exporte plus qu'auparavant, l'accroissement de son revenu consécutif à la hausse des exportations va provoquer une augmentation des importations; il existe, en effet, une propension marginale a importer tout a fait analogue à la propension marginale à consommer.

Alors que le mécanisme d'adaptation chez les classiques tend toujours vers l'équilibre, il n'en est pas de même chez les keynésiens ; il est tout à fait possible, dans certaines conditions, qu'un déséquilibre persiste, en raison de l'inégalité de ce qu'on a appelé les effets induits. En effet, une balance commerciale favorable agit sur l'économie d'un pays comme un investissement. En d'autres termes, les revenus engendrés par le mouvement favorable de la balance commerciale sont supérieurs au montant de la balance commerciale. La propension marginale à importer est nettement inférieure à l'unité et représente donc une fraction des revenus engendrés par la balance commerciale. Il n'y a pas de raisons de croire que l'accroissement des importations sera aussi considérable que l'accroissement des exportations. D'autre part, l'accroissement des importations va accroître le revenu d'un autre pays qui à son tour va augmenter, par le truchement de sa propension à importer, ses achats dans le premier pays. Mais ces répercussions induites n'assurent pas un équilibre stable de la balance des comptes. ${ }^{22}$

22 Ces quelques idées ne sont qu'un schéma des mécanismes ingénieux développés depuis quelques années. On en trouvera une description plus précise dans la remarquable rétrospective de la théorie du commerce international publiée par Lloyd-A. Metzler dans le Survey of Contemporary Economics, op. cit. 
Puisqu'une variation de la balance commerciale a des effets assimilés à une variation de l'investissement intérieur, un pays doit se protéger contre une balance commerciale défavorable qui risquerait d'amorcer une baisse de la demande effective. Cette protection ne peut être que volontaire puisqu'il n'y a pas de retour automatique à l'équilibre. Quelles mesures doivent alors être prises ?

La dévaluation semblait évidemment être une solution. Mais plusieurs influences se conjuguaient pour limiter l'espoir d'obtenir des résultats par ce moyen. Les dévaluations des années d'avant-guerre n'avaient pas été très heureuses, dans l'ensemble. On avait même remarqué que certains pays qui avaient pu pratiquer une dévaluation sans représailles de la part des autres pays n'avaient pas toujours obtenu l'accroissement des exportations que l'on en espérait. D'autre part les post-keynésiens, étudiant les élasticités aux prix de la demande et de l'offre des produits sur les marchés internationaux, en étaient arrivés à la conclusion suivante : si l'on suppose que les élasticités de l'offre sont infinies, comme elles le sont souvent sur la courte période pour les produits manufacturés, il suffit pour qu'une dévaluation réduise le déficit d'une balance commerciale entre deux pays, que la somme des élasticités de la demande d'importations dans ces deux pays soit supérieure à l'unité. 23

Or, dans les années qui suivirent la guerre, de nombreuses études empiriques (celles de Hinshaw et de Tse Chun Chang en particulier) en arrivent toutes à la même conclusion : les élasticités de la demande sont très faibles. 24

Alors est apparu ce qu'on a appelé depuis, le «pessimisme des élasticités ». Non seulement, disait-on, une dévaluation n'aide pas né-

23 A.-P. Lerner, The Economics of Control, MacMillan, New-York, 1944, pp. 377 et s.

24 Randall Hinshaw, "American Prosperity and the British Balance of Payment Problem" dans Review of Economic Statistics, février 1945, p. 4. Tse Chun Chang, Cyclical Movements in the Balance of Payments, Cambridge University Press, 1951. Les premiers résultats des travaux de Tsé ont été publiés dans diverses revues à partir de 1946. 
cessairement à résorber un déficit de la balance des comptes, elle peut même l'accentuer. Le pessimisme des élasticités s'est d'ailleurs communiqué à d'autres secteurs de la théorie. Les tarifs, par exemple, devenaient une arme peu efficace pour se défendre contre des entrées surabondantes d'importations. En définitive, la seule forme de contrôle vraiment utile à court terme dans certaines conditions, ne pouvait être que la restriction quantitative des marchandises et du change. On jugeait souvent que les restrictions étaient nocives en empêchant une distribution internationale rationnelle de la production. On ne croyait pas, dans de nombreux cas, pouvoir les éviter.

Sans doute tous les post-keynésiens n'ont pas poussé aussi loin le raisonnement. Et surtout, tous n'ont pas accepté de souscrire aux simplifications proprement invraisemblables qu'il fallait poser pour en arriver là. Il reste cependant que l'influence de ce pessimisme des élasticités a été profonde, et les conclusions les plus radicales de l'école post-keynésienne ont pu servir de prétextes à une politique que les États, après la guerre, pratiquaient avec vigueur.

En 1950, cependant, un article d'Orcutt remettait en cause les conclusions élaborées par l'école la plus avancée des postkeynésiens. ${ }^{25}$ Le principe avancé par Orcutt était simple : les méthodes mathématiques utilisées pour calculer les cœfficients d'élasticité tendent a réduire systématiquement la valeur des coefficients. L'article eut un retentissement considérable. Déjà avant sa publication, on avait eu tendance à revenir sur les idées nouvelles et à les réexaminer. 26 Orcutt donna le coup de grâce à bien des illusions.

Le revirement d'ailleurs arrivait à son heure. Les abus des contrôles et la complexité des réglementations dans le commerce international cédaient progressivement devant les efforts systématiques de libéralisation de la part des organismes internationaux et de certains gouvernements qui n'avaient plus intérêt à la poursuite des restrictions.

25 G.-H. Orcutt, "Measurement of Price Elasticities in International Trade, Review of Economic Statistics, mai 1950.

26 Voir en particulier le livre de Jacob Viner, International Trade and Economic Development, Oxford University Press, 1953, pp. 65 et s. 
Il ne faudrait pas conclure de cet échec retentissant que la théorie keynésienne du commerce international a reçu un démenti formel. Dans ce domaine, comme dans celui de l'analyse économique générale, le keynésianisme a lancé des concepts utiles et a attiré l'attention sur des relations économiques qui n'avaient pas été précisées. L'aventure du théorème des élasticités critiques illustre, cependant, l'imprudence extrême de certains post-keynésiens. Elle remet en question la liaison de la micro-économie et de la macro-économie délaissée par de nombreux théoriciens, mais qui est inéluctable après les abus des dernières années.

\section{Le retour au classicisme monétaire}

$\underline{\text { Retour à la table des matières }}$

L'épanouissement des théories post-keynesiennes, leur pénétration au niveau des politiques économiques ont été depuis quelques années contenus puis remis en cause par ce que l'on peut toujours considérer comme un retour en arrière, mais qui représente un point tournant décisif de la politique gouvernementale, c'est-à-dire la réapparition de la politique monétaire. Déjà au moment de la guerre de Corée, on avait pu constater aux États-Unis un assouplissement des conditions monétaires. l'État n'était plus disposé à assurer un approvisionnement infini de monnaie à des taux très bas. La récession de 1954 marque aux États-Unis et au Canada un retour à des formes de contrôles de la conjoncture disparue depuis la crise. On recommence, au niveau des études théoriques, à s'interroger sur le sens d'une politique monétaire, et le classicisme d'antan refleurit. Après des années d'oubli, les techniques d'autrefois de manipulation du marché monétaire et du marché financier, parfois aussi des mouvements internationaux de capitaux sont redécouvertes. Lorsque la Banque d'Angleterre pour faire face à des menaces d'inflation et au déficit de la balance des paiements, porte son taux d'escompte à 7 pour cent, on peut considérer qu'un chapitre de l'histoire des doctrines économiques est clos. Lorsque cette mesure attire a Londres, comme autrefois, des capitaux étrangers en quête de placements, il n'est pas faux de dire que l'histoire des faits économiques vient de s'engager dans une nouvelle voie. De tels exemples sont 
des symboles, sans doute, mais des symboles de faits et de politiques qui sont apparus un peu partout dans le monde.

Les raisons de ce retour en arrière sont multiples. Des ambiguïtés sont disparues. Ainsi l'identité de certains objectifs de la doctrine postkeynésienne et des partis socialistes à l'égard de la sécurité sociale s'est dissoute lorsque, dans un grand nombre de pays du monde, les gouvernements, souvent de gauche après la guerre, ont glissé vers la droite. De même le dirigisme d'après-guerre imposé par l'anormalité de l'économie mondiale, et qui s'accommodait si bien d'une politique conçue comme étant la manipulation de quantités globales, a-t-il été rejeté au fur et à mesure que les économies nationales sortaient du marasme.

Enfin quinze années de hausse de prix ont fini par laisser une trace psychologique profonde et la lutte contre l'inflation est devenue d'autant plus violente qu'on n'en voyait plus la nécessité ou l'utilité. Dans ces conditions, les Banques centrales retrouvaient et leur rôle et leurs pouvoirs.

D'ailleurs la structure fiscale se prêtait d'autant plus mal à la lutte contre l'inflation, que celle-ci devenait, en temps de paix, davantage larvée qu'ouverte, et, qu'après des années d'impôts très élevés et de dépenses de guerre chaude ou froide, l'opinion publique résistait fortement à une hausse des impôts, fut-elle conjoncturelle. How to Pay for the War ne pouvait plus dix ans après la guerre devenir how to pay for peace.

Élaborés pendant la crise, appliqués pendant la guerre, modifiés pendant les années qui la suivirent, le keynésianisme et ses prolongements, s'identifiaient peut-être avec l'anormal, tout au moins avec le critique. Les nuances théoriques et politiques reprenaient leurs droits, avec le retour à l'économie « paisible ».

Dans une situation si nouvelle, le corps de théories que l'on peut caractériser comme étant post-keynésien a achevé de se dissoudre. Les modèles macro-économiques d'autrefois, où la monnaie ne jouait aucun rôle, cessent d'être les cadres simplifiés d'expérience de la politique économique. Quand économistes et gouvernement recommencent 
à discuter des types d'inflations ; lorsqu'à nouveau, l'on s'interroge sur la vitesse de circulation de la monnaie ; lorsque, enfin, l'intérêt porté au niveau global de la demande effective cède la place à l'étude des substitutions automatiques des activités à la suite d'une modification du niveau de la masse monétaire, alors peut-on dire que la théorie keynésienne ou post-keynésienne a relâché l'emprise qu'elle exerçait sur la politique.

On aurait tort cependant de croire qu'il ne reste rien de la théorie ou de la doctrine. jamais plus un gouvernement ne relèvera-t-il ses impôts en période de récession, comme on l'avait fait si souvent au début de la Grande Crise. Dans ce domaine comme dans bien d'autres les idées de Lord Keynes et de ses disciples, certains de leurs concepts et de leurs techniques sont entrés dans les moeurs. Une partie de l'enseignement a été complètement assimilée non pas seulement par les théoriciens mais par le public.

Il n'y a plus à proprement parler de politique économique keynésienne. Ce qu'il en reste cependant continuera longtemps encore d'influencer, inconsciemment souvent, l'attitude des autorités gouvernementales.

Fin du texte 